\title{
Análise do Comportamento Dinâmico de uma Suspensão Trailing Arms a ser aplicada em um Perfilômetro Inercial Rebocável
}

\author{
Alessandro Borges de Sousa Oliveira (UnB/FGA) \\ Rita de Cássia Silva (UnB/FGA) \\ Malthus Alves Rodrigues (UnB/FGA)
}

Universidade de Brasilia- UnB/FGA

Resumo: o trabalho visa a apresentar a concepção de uma suspensão trailing arms aplicável a um perfilômetro inercial rebocável. Sendo rebocável, o equipamento necessitará do seu próprio sistema de suspensão devendo assegurar ao módulo de medição um comportamento inercial. São utilizados softwares de análise multicorpos, de elementos finitos, além do conceito de transmissibilidade. $\mathrm{Na}$ Engenharia, esses equipamentos são imprescindíveis na aquisição de sinais de pista, que configuram a maior fonte de excitação das suspensões veiculares. Tendo grande impacto no estudo de ciclo de vida de elementos automotivos e, consequentemente, na segurança veicular. Os resultados mostram que o uso de ferramentas numéricas e aplicação dos conceitos é adequado no desenvolvimento da suspensão, obtendo-se um sistema de suspensão com baixa transmissibilidade.

Palavra chave: perfilômetro, suspensão, perfil de pista

\begin{abstract}
: this work aims to present the design of a trailing arms suspension of an inertial profiling system. As the setup need its own suspension system, it needs to be able to ensure an inertial behavior to the measuring module. Multibody dynamic and finite element software are used, also considering the transmissibility concept. These equipment are essential to determine the irregularities of road profiles, which represent the greatest source of excitation in vehicle dynamics having an impact on the life cycle of automotive elements and, consequently, on the safety. The results show that numerical tools and dynamic concepts are suitable to suspension's development furnishing a subsystem with low transmissibility.
\end{abstract}




\section{1 - Introdução}

Segundo dados do DNIT (2015), o Brasil possui uma malha rodoviária de aproximadamente 1,72 milhão de quilômetros de estrada, sendo que apenas $12,2 \%$, o equivalente a $210.618 \mathrm{~km}$ é pavimentado. Devido à instalação das montadoras automotivas e dos incentivos físcais dados pelo governo ao transporte rodoviário na década de 50,60 \% da produção nacional é escoada pelas rodovias, o que as torna um elemento vital na economia do país, conforme apontado por Barella (2008).

Manter estradas em bom estado de conservação tem sido um grande desafio para os governos federal e estaduais. Fatores como o escoamento da água; a qualidade dos materiais utilizados para construção; veículos de carga com tara acima da permitida por lei, dentre outros influenciam na degradação das rodovias.

De fato, durante o tráfego por um pavimento com imperfeições e ondulações, o veículo é submetido a excitações externas provenientes, principalmente, das irregularidades da pista, que resultam em um carregamento dinâmico atuando sobre o veículo, sobretudo no subsistema suspensão. Tal carregamento, se registrado, traduz-se em um sinal de aceleração vertical $\left(\mathrm{m} / \mathrm{s}^{2}\right)$.

Do ponto de vista da avaliação do ciclo de vida de elementos mecânicos, ter a suspensão veicular submetida a carregamentos dessa natureza leva a um impacto direto na vida útil dos componentes mecânicos desse subsistema e, consequentemente, na Segurança Veicular, conforme afirmam Barella (2008) e Rodrigues (2015).

Embora, parta-se do senso comum que as irregularidades de uma pista sejam apenas os buracos nas estradas nacionais, a definição formal é mais ampla: qualquer elevação vertical, em relação a um plano perfeito, é considerada uma irregularidade. Assim, desde uma elevação micrométrica até um buraco visto a olho nu trata-se de uma irregularidade. No entanto, o cenário ideal seria que a rodovia fosse perfeitamente plana, sem qualquer tipo de rugosidade. Entretanto, na prática esta condição ideal é utópica, conforme citado por Côrtes (2009).

A norma DNER PRO 164/94 conceitua as irregularidades de pavimentos como: “O desvio da superfície da rodovia em relação a um plano de referência, que afeta a dinâmica dos veículos, a qualidade do rolamento e as cargas dinâmicas sobre a via". Tal definição se impõe por dois aspectos a dinâmica do veículo (longitudinal, transversal e vertical) e sobre a conservação da via.

Sayers e Karamihas (1998) destacam, em seu trabalho, que seria infactível medir a irregularidade de toda a área de uma pista, sendo preferível fazê-lo em um dado trecho entre dois pontos. Esse trecho representaria uma amostra local denominada perfil de pista medida com base em um plano perpendicular à superfície "cortando" o pavimento, conforme ilustrado na Fig.(1). Sayers e Karamihas (1998) e Côrtes (2009) apresentam o perfil de pista como uma combinação de ondas em um amplo espectro de frequências. Considerando o perfil da superfície, há a divisão em perfil de rugosidade e 
perfil de ondulação. O primeiro é composto por ondas de alta frequência, enquanto o segundo por ondas de baixa frequência, conforme esquema da Fig.(1). Destaca-se que a denominação completa seria perfil de pista longitudinal, em que o termo "longitudinal" refere-se ao eixo em que há o tráfego principal dos veículos (Sayers e Karamihas (1998)). A Fig. (2) destaca o sentido longitudinal de medição do perfil de pista.

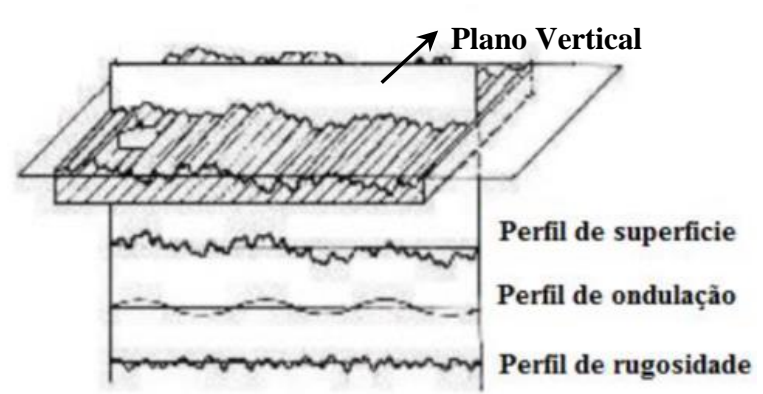

Figura 1 - Composição do perfil de superfície de uma pista: perfis de ondulação e rugosidade (Fonte: Côrtes, 2009)

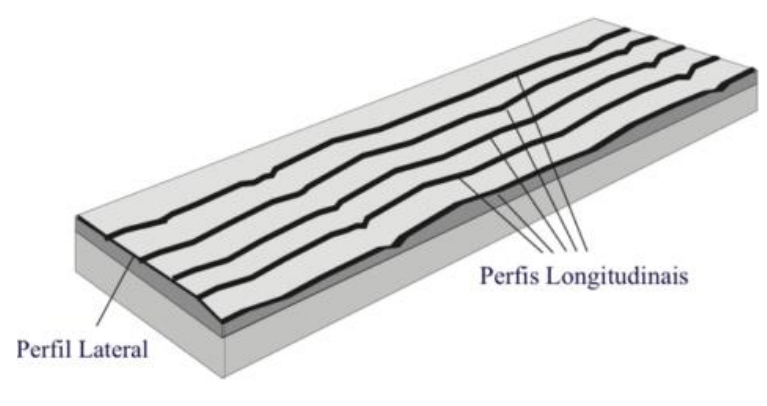

Figura 2 - Orientação de perfis: transversal e longitudinal (Fonte: Adaptado de Sayers e Karamihas,1998)

Diante do exposto, a obtenção do perfil de pista longitudinal se torna essencial em estudos que envolvam determinação do ciclo de vida e índices de confiabilidade $(\beta)$ de componentes automotivos e definição do perfil e frequência de atuação de certos níveis de carregamento em pontos específicos do subsistema suspensão. Dessa maneira, o perfil de pista servirá de input para simulações numéricas CAE (Computer Aided Design) em ambiente multicorpos ou em elementos finitos, além de estudos experimentais visando à Segurança Veicular. Isto porque eles representam o carregamento real a que o subsistema está submetido, Freitas (2006) e Rodrigues (2015).

Embora, haja perfis de pista experimentais disponíveis (Belgian Pavé, ISO 8016 ou em softwares comerciais) estes não representam adequadamente a malha viária brasileira, sendo mais representativos de pistas europeias e americanas, Vieira (2011). Um dos dispositivos capazes de estabelecer o perfil da superfície da pista é o perfilômetro inercial (seção 2). Ele utiliza um sensor de distância a laser e um acelerômetro montado em um veículo de testes que trafega na pista, segundo a velocidade da via e registra, através do acelerômetro, o espectro de aceleração vertical da massa suspensa (carroceria) ao longo de um trecho longitudinal. 

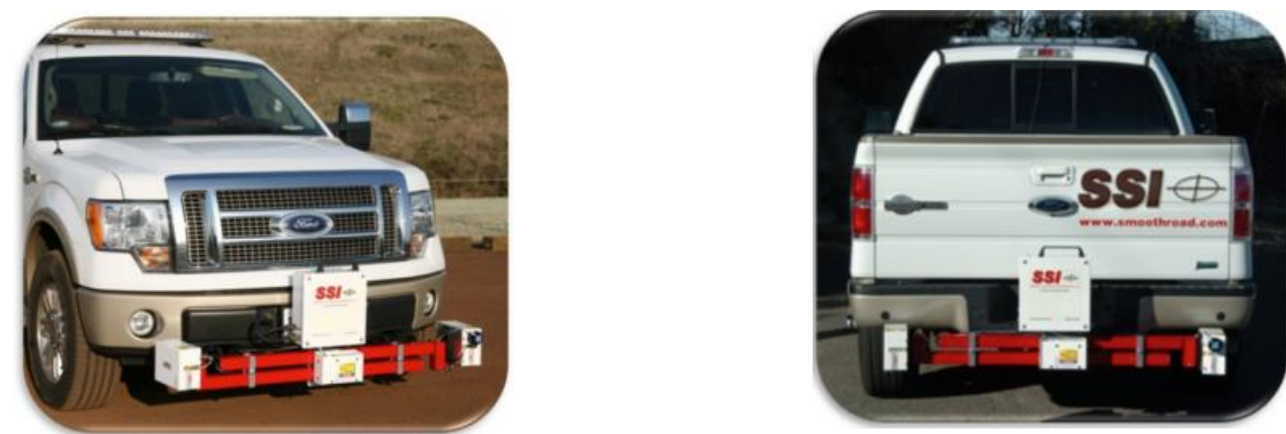

Figura 3 - Exemplos de fixação de perfilômetros inerciais (Fonte SSI ${ }^{\circledR}$ ) a) fixação na dianteira b) fixação na traseira

Empresas como a americana SSI ${ }^{\circledR}$ fabricam perfilômetros inerciais com diferentes configurações, como mostrado na Fig. (3).

Diante desse contexto, o presente trabalho vem contribuir apresentando a proposta de um perfilômetro inercial rebocável com dois eixos independentes e suspensão trailing arms nas quatro rodas. Ressalta-se que enfoque do presente trabalho se dá na etapa de simulação numérica em softwares CAE (ADAMS/View ${ }^{\circledR}$ e Ansys ${ }^{\circledR}$ workbench 16) do sistema de suspensão do veículo rebocável. Para tanto, uma análise em dinâmica vertical de 1/4 de veículo será efetuada em ambiente multicorpos (ADAMS/View®). Nessa etapa, é necessário considerar o conceito de transmissibilidade, que será brevemente tratado no item 3. Os modelos em ambiente multicorpos e elementos finitos encontram-se no item 4.

Destaca-se que a construção do veículo rebocável, instalação da instrumentação, teste e validação representam os trabalhos futuros e, tal iniciativa proverá o grupo de um equipamento capaz de avaliar o perfil de pista de pavimentos na região, aprimorando os métodos de pesquisa em Segurança Veicular.

\section{2 - Abordagem geral sobre perfilômetros}

O primeiro equipamento para avaliação de pavimentos, segundo Gillespie (1992b), foi o Viagraph (Fig. 4), logo após o surgimento do veículo à combustão interna. Com o aumento da velocidade dos veículos e a necessidade de estudos mais aprofundados em segurança e dinâmica veicular desenvolveuse o Rolling Straightedge, que foi precursor do perfilógrafo (Fig. 5). Para essa nova geração de equipamento, o deslocamento se dava por rodas e não mais arrastado como o Viagraph.

O funcionamento do perfilógrafo é semelhante ao do Viagraph. Uma roda auxiliar e independente das responsáveis por seu deslocamento, passa pelas irregularidades marcando o deslocamento vertical da régua ligada a ela. 


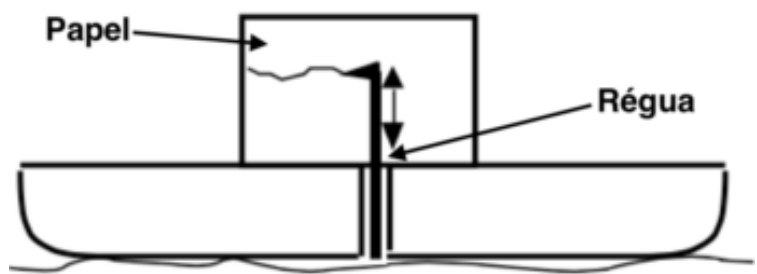

Figura 4 - Esquema do Viagraph (Fonte: Adpatado de Sayers e Karamihas, 1998)

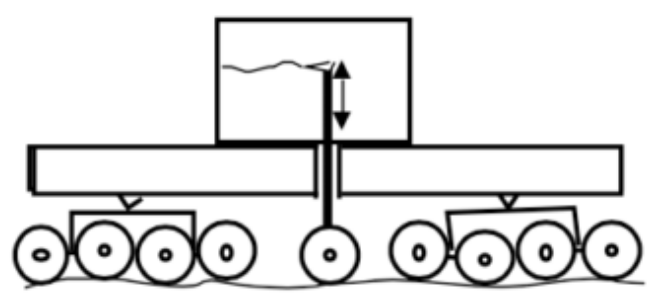

Figura 5 - Esquema simplificado do perfilógrafo ((Fonte: Sayers e Karamihas, 1998)

Já na década de 60 com a disponibilidade de melhor tecnologia de medição, a verificação passou a ser diretamente no veículo de teste com auxílio de um transdutor e, os resultados eram processados por um contador montado no veículo. Estes equipamentos são classificados como medidores do tipo resposta.

Sayers e Karamihas, (1998) apontam que nos anos 80, os medidores do tipo resposta eram maioria na medição de irregularidades de pavimentos até a popularização dos perfilômetros inerciais. No Brasil a norma DNER PRO 164/94 regula os procedimentos de medição para este tipo de equipamento.

\section{1 - Perfilômetro inercial}

Segundo Sayers e Karamihas (1998) três elementos são necessários para se medir irregularidades: uma elevação de referência, a altura relativa à referência e distância longitudinal ao longo do trecho estabelecido. As variações de elevação verticais $(\mathrm{mm})$ em relação à elevação de referência, estabelecida ao longo da distância longitudinal, constituem o perfil de pista longitudinal. A elevação de referência corresponde à distância vertical entre o ponto de instalação do sensor de distância a laser na carroceria e a pista. No caso do perfilômetro, considera-se ainda um referencial inercial.

Desenvolvido pela GM na década de 60, os perfilômetros inerciais, utilizam um sensor de distância a laser e um acelerômetro, para obter um perfil de pista longitudinal. Estes equipamentos são montados em um veículo de testes, que trafega na velocidade normal da via.

Para o perfilômetro, conforme Hu (2006) e Barella (2008), a distância medida pelo sensor de distância a laser será diferente da altura do referencial inercial, devido às irregularidades na pista, indicadas na Fig. (6).

Portanto, as irregularidades do pavimento para um dado trecho será a subtração entre o valor constante do referencial inercial (elevação de referência) e a medida obtida pelo sensor de distância a laser. Caso o veículo não sofra nenhum deslocamento vertical essa distância seria constante.

No entanto, como o automóvel de teste está trafegando no pavimento, os deslocamentos verticais da carroceria do veículo, devido às irregularidades da pista, modificam a elevação de referência. Essa modificação de referencial é corrigida com a instalação de um acelerômetro em conjunto com o sensor 
de distância a laser. Segundo Hu (2006), este sensor registrará as acelerações verticais na carroceria. Barella (2008) ressalta que o acelerômetro deve ser acoplado em perfeito alinhamento com o sensor de distância para que ambos possam gerar informação na mesma frequência.

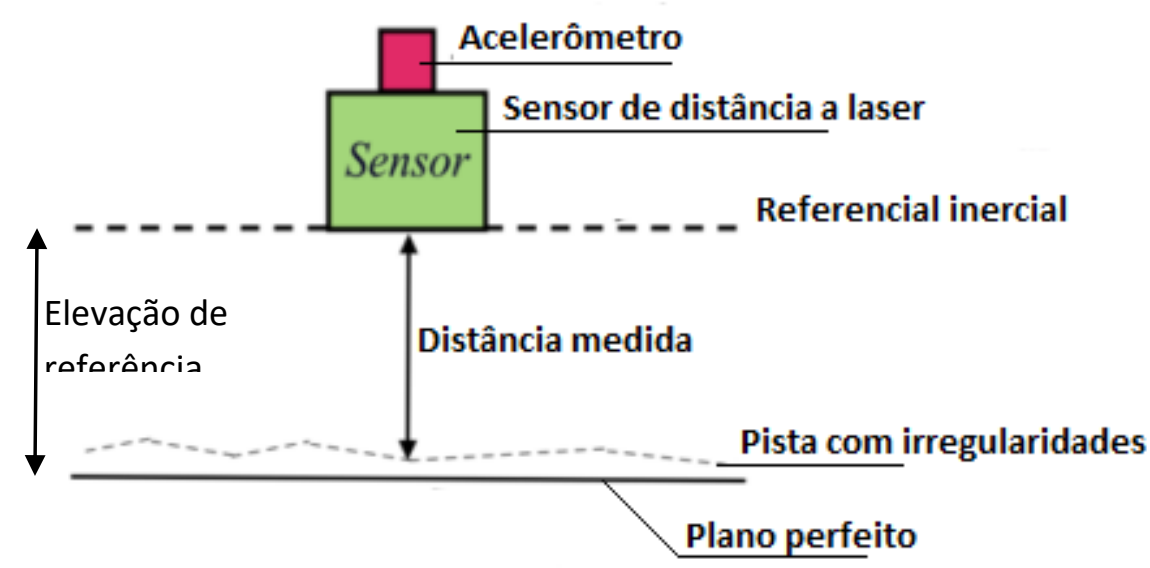

Figura 6 - Ilustração de medição do perfilômetro inercial

Com o sinal do acelerômetro, efetuando-se dupla integração determina-se o deslocamento da carroceria e se calcula, assim, a elevação de referência para cada instante. Em seguida, o perfil longitudinal de pista será a subtração entre o referencial inercial corrigido, pelo sinal do acelerômetro, e as medições efetuadas pelo sensor de distância a laser.

A Fig. (7) traz um protótipo de perfilômetro desenvolvido por Barella (2008), adaptado nas partes traseira e dianteira de veículos de diferentes modelos, portanto com diferentes comportamentos em dinâmica veicular, ou seja, massas distintas, rigidez de mola, coeficiente de amortecimento, geometria de suspensão.
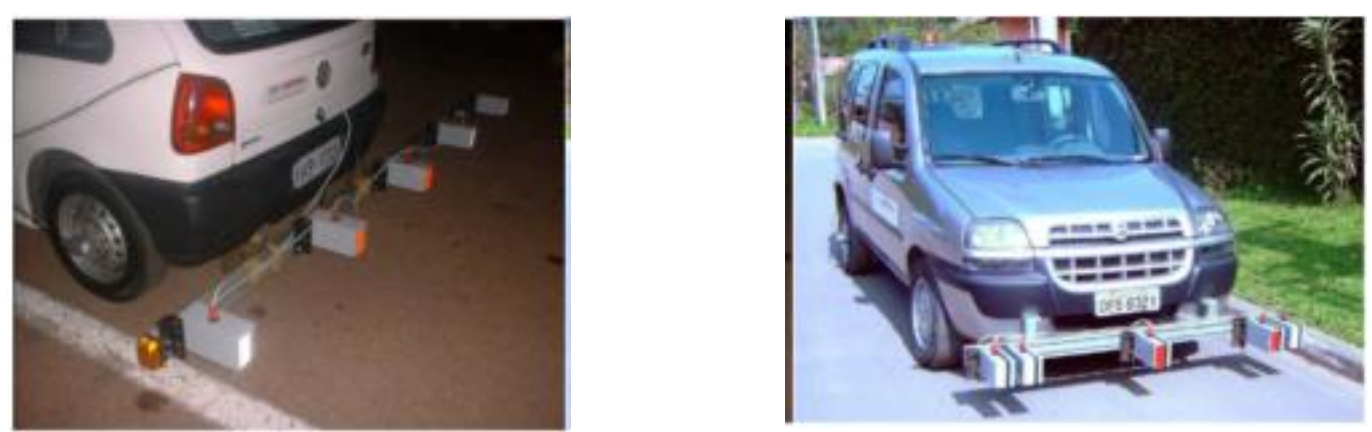

Figura 7 - Modelo de perfilômetro inercial (Fonte: Barella (2008))

\subsection{1 - Apresentação do perfilômetro inercial rebocável}

A ideia do perfilômetro inercial rebocável difere-se do tratado em 2.1.1, porque o módulo de medição será acoplado e rebocado, durante o teste. Sendo assim, em seu projeto o reboque terá um 
sistema de suspensão capaz de isolar ao máximo a carroceria, onde se aloja o referido módulo, das irregularidades do pavimento.

No mercado, um modelo de perfilômetro inercial rebocável é o Lightweight Profiler da SSI®, Fig.(8a). Em relação a esse modelo, a presente proposta apresentará dois diferenciais: a montagem com dois eixos e suspensão trailing arms nas quatro rodas. Outro ponto relevante é que o dispositivo contará com molas pneumáticas por oferecem uma faixa de pressão, que permite alterações na rigidez. O reboque tem dimensões de $2 \mathrm{~m}$ de comprimento com 1,4 m de largura com uma carroceria com 300 mm de altura (Fig. 8b).
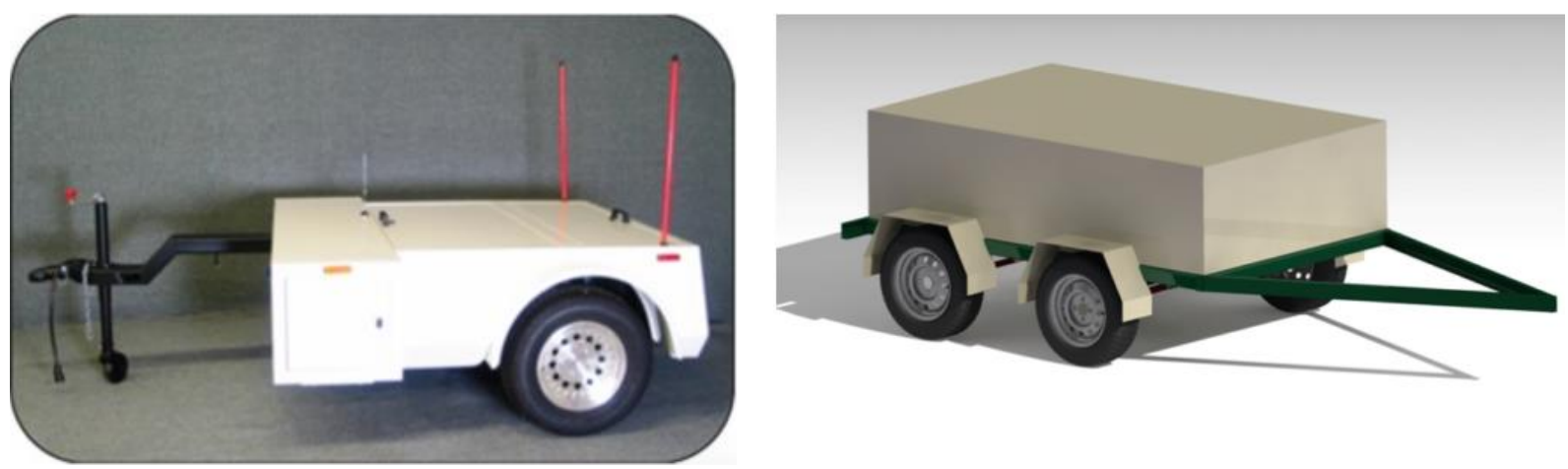

Figura 8 - Modelos de perfilômetros inerciais rebocáveis a) Modelo Rebocável Lightweight Profiler (Fonte: SSI $\left.{ }^{\circledR}\right)$ b) Proposta do perfilômetro rebocável

O módulo de medição consiste de um sensor de deslocamento de CCD de alta velocidade e alta precisão, modelo GM3000 da Keyence. Este sensor é acoplado a uma cabeça medidora de longa distância de ponto pequeno modelo LK-G152. Outra característica observada acerca das carrocerias tradicionais é que estas são fabricadas de materiais metálicos. Para a presente proposta há a demanda de manter uma temperatura adequada para os sensores que está na faixa entre 0 e $50^{\circ} \mathrm{C}$. Para isto será utilizado um material compósito com núcleo de poliestireno. Essa alternativa garante simultaneamente o isolamento da temperatura externa e a rigidez esperada para a carroceria de um veículo de reboque.

\section{3 - Aspectos gerais da transmissibilidade na dinâmica vertical}

Conforme apresentado por Gillespie (1992a) e Sayers e Karamihas (1998), o estudo da dinâmica vertical se baseia na compreensão do comportamento veicular ao ser exposto a fontes de excitação. A condição inercial do sistema de medição está diretamente ligada aos níveis de acelerações verticais e vibrações transmitidas ao módulo, assim como definir as fontes de excitação, que atuam no veículo. Ao se expor a fontes de excitação, o veículo age como um filtro gerando uma resposta dinâmica, que é caracterizada pela relação entre as entradas (perfil de pista) e saídas (deslocamento vertical da carroceria). A razão entre as excitações de saída pelas excitações de entrada é denominada transmissibilidade. Nesse caso, a suspensão é o sistema isolador, que atenua o movimento transmitido 
ao sistema, ou seja, na massa suspensa (carroceria). A Fig. (9) apresenta a metodologia de estudo da dinâmica vertical, segundo Gillespie (1992a).
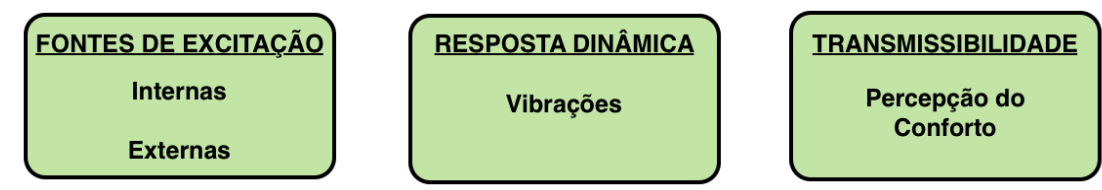

Figura 9 - Etapas para o estuda da dinâmica vertical. (Fonte: Adaptado de Gillespie (1992a))

O perfil de pista é composto por ondas de baixa frequência que provocam movimento de corpo rígido. Esta zona, denominada de ride primário, está localizado na faixa de frequência entre $1 \mathrm{~Hz}$ a 5 Hz. A faixa de 5 a $100 \mathrm{~Hz}$ é denominada de ride secundário e é responsável pelas vibrações e acelerações verticais mais severas que, embora tenham amplitudes inferiores ao ride primário; a frequência das vibrações na carroceria é maior provocando uma grande faixa de excitação do módulo de medição (Fig. 8b).

Assim, esse trabalho traz a concepção do sistema de suspensão de um perfilômetro inercial rebocável focando na determinação da transmissibilidade de vibrações da excitação provocada pelo perfil de pista à carroceria do módulo de medição (Fig. 8b).

\section{4 - Métodos}

Nessa seção, baseado no exposto nas seções 2.1.1 e 3, serão tratados os métodos de simulação empregados para análise de $1 / 4$ de suspensão a ser empregada no perfilômetro inercial rebocável apresentando a metodologia adotada para alcançar os objetivos do trabalho.

\section{1 - Modelagem do sistema de suspensão do perfilômetro inercial em ambiente multicorpos}

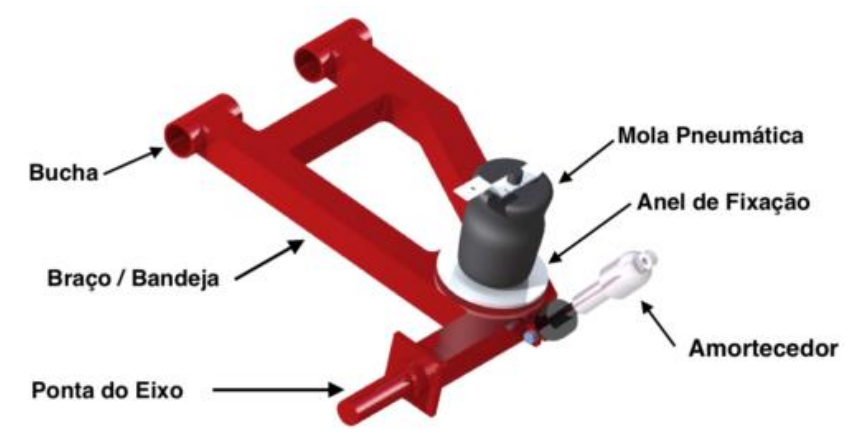

(a)

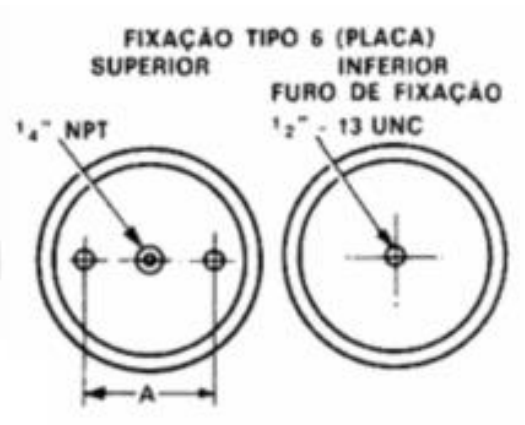

(b)

Figura 10 - Suspensão trailing arms do perfilômetro inercial rebocável a) modelo CAD b) anel de fixação da mola (Fonte: http://www.niagara.com.br/v2/comercial/firestone01.htm)

A proposta preliminar baseou-se em design de braços de suspensão trailing arms do mercado, assim como materiais e dimensões. Foram utilizados tubos de aço quadrado 40x40 com 2 mm de 
espessura, mola pneumática juntamente com anel de fixação, amortecedor e buchas. O anel de fixação será soldado ao braço de controle e, em seguida, a mola será parafusada a este anel, para fixá-la ao braço. A Fig. (10) apresenta a suspensão em CAD e o anel de fixação superior e inferior, conforme dados do fabricante. A construção do modelo em ambiente multicorpos inicia-se pela importação das coordenadas dos keypoints (Fig. (11)). Os keypoints são os pontos de interesse para modelagem do braço no sistema multicorpos que são: o centro de massa indicado pela letra (A); os pontos de fixação do braço no chassi indicados pela letra (B) e o ponto onde o conjunto mola -amortecedor será montado, indicada pela letra (C) (Fig. (11)).

Juntas são elementos que garantem a conexão entre os corpos, influenciando o movimento relativo entre eles; restringindo ou liberando graus de liberdade. Relativamente às juntas aplicadas ao modelo, tem-se que para unir o braço de suspensão (2 em Fig. 13) à massa suspensa (3 em Fig. 13) utilizou-se uma junta revoluta (A em Fig. 12). A junção do braço de suspensão (2 em Fig. 13) à roda (1 em Fig. 13) usou-se uma junta fixa. A última junção é entre a massa suspensa (3 em Fig. 13) e o ground. Nessa união usou-se uma junta inplane (C em Fig. 12) e parallel axis (D em Fig. 12).

Em seguida, para inserção do pneu à ponta de eixo do modelo (Fig. 12 e 13) usou-se o módulo ADAMS/Tire®. Foram inseridas as características da mola pneumática e do amortecedor (Fig. 10a), através de curvas força x deformação $(\mathrm{mm})$ e força $x$ taxa de deformação $(\mathrm{mm} / \mathrm{s})$.

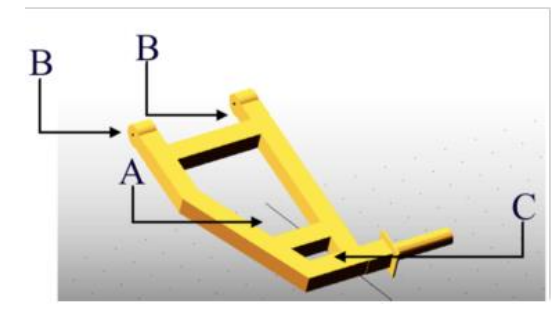

Figura 11 - Posição dos keypoints

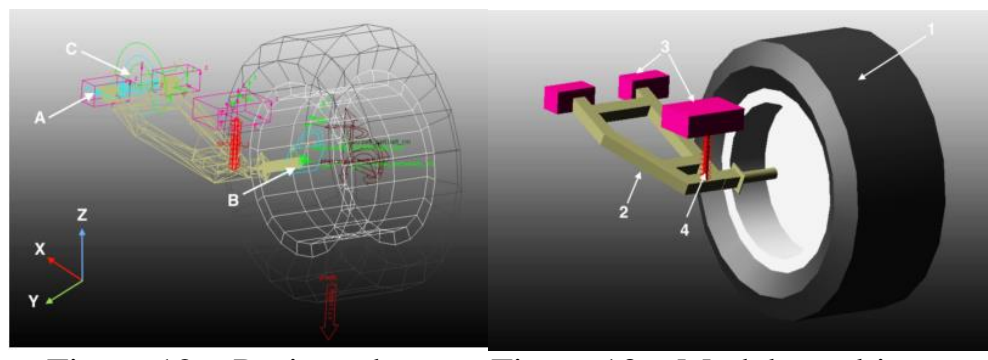

Figura 12 - Posição das juntas aplicadas ao modelo
Figura 13 - Modelo multicorpos do $1 / 4$ de suspensão

Para excitação do modelo multicorpos foi aplicado o perfil de pista extraído do Carsim®, Fig. (14), mediante a inserção de três obstáculos pontuais denominados tachões de $50 \mathrm{~mm}$ de altura. Tais obstáculos foram inseridos a 9, 90 e 119 m ao longo do perfil (ver setas em Fig. 14). No entanto, a fim de verificar os deslocamentos da massa suspensa (item 3 em Fig. 13) ocasionados pelas irregularidades de altas frequências, perfil de rugosidade (ride secundário - seção 3), filtrou-se o sinal em Fig. 14 com um filtro moving average, Fig.15. Observa-se que as ondas com grandes comprimentos, foram atenuadas, Fig. 15. 


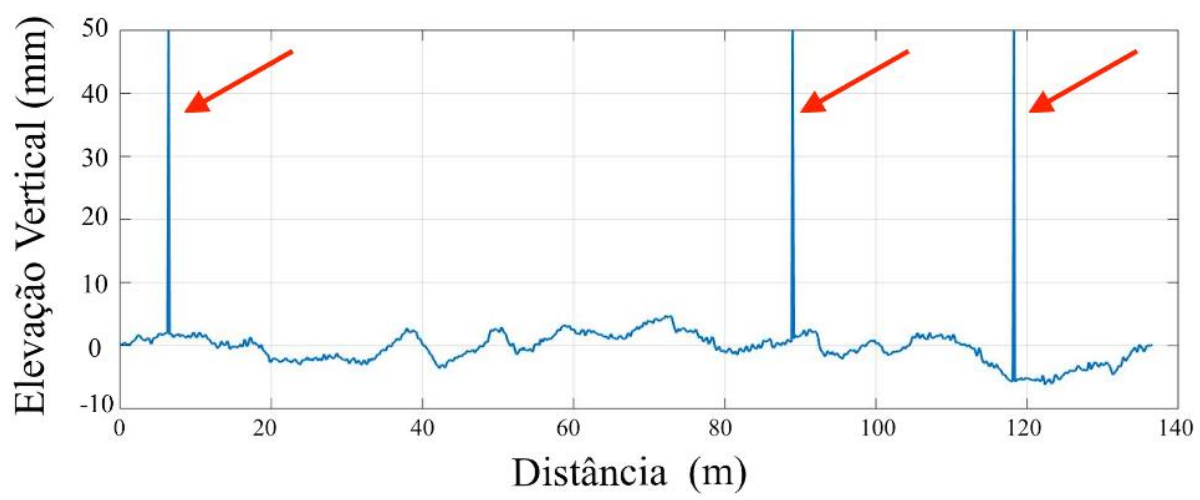

Figura 14 - Perfil de pista de excitação sem filtragem com tachões

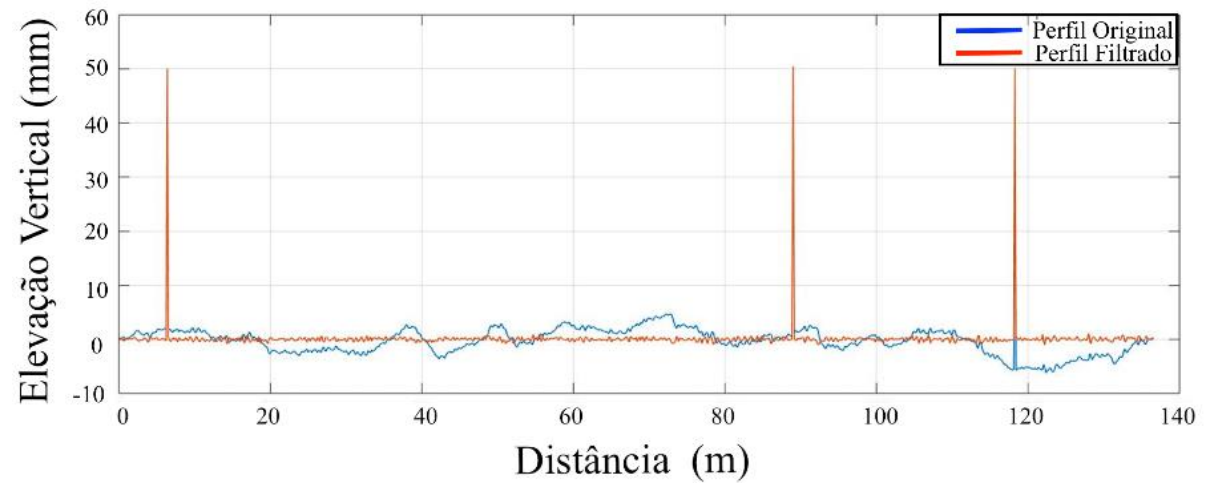

Figura 15 - Comparação entre perfil de pista com tachões original e filtrado

Sabe-se que o objetivo principal do sistema de suspensão é diminuir a transmissibilidade (seção 3) entre a pista e o módulo de medição (Fig. 8b), mantendo a massa suspensa inercial. Logo, o modelo multicorpos auxiliará na verificação desses deslocamentos ao trafegar pelos perfis mostrados em Fig. 15 (original e filtrado).

\section{2 - Modelagem em elementos finitos}

O modelo em elementos finitos possui 19.334 elementos tetraédricos de $8 \mathrm{~mm}$, após estudo de sensibilidade de malha. Da análise realizada em 4.1 com o perfil de pista original (Fig. 14) sem tachões, foram estabelecidos os carregamentos atuantes nos pontos de fixação do braço ao chassi, no ponto de fixação da mola pneumática e na ponta de eixo. Da análise a bandeja apresentou um estado de tensões com magnitude inferior à tensão de escoamento do material adotado $280 \mathrm{MPa}$ utilizando-se o Critério de escoamento de Von Mises. A Tab. (1) mostra os valores definidos e local de aplicação.

\section{5 - Resultados e discussão}

De uma combinação ideal entre pressão da mola pneumática, valor em $\mathrm{kg}$ da massa suspensa e curva de amortecimento, monitorou-se a amplitude do primeiro pico (A), amplitude do segundo pico (B), valor rms (C) e tempo de atenuação (D), conforme Fig. (16) que representa o deslocamento 
vertical da massa suspensa do perfilômetro inercial rebocável (3 em Fig.13). Essa análise foi realizada a partir de um planejamento fatorial, segundo Montgomery (1997).

Tabela 1 - Forças atuando nos pontos sinalizados

\begin{tabular}{|c|c|c|c|}
\hline Carregamento & Valor & Local de atuação & Pontos de fixaçáa do \\
braço no chassi \\
\hline Momento em X & 5.740 N.mm & Ponta do eixo \\
\hline Momento em Y & -111 N.mm & Ponta do eixo \\
\hline Momento em Z & 232.000 N.mm & Ponta do eixo \\
\hline Força em X & $-1.576 \mathrm{~N}$ & Ponta do eixo \\
\hline Força em $\mathrm{Y}$ & $1.231 \mathrm{~N}$ & Ponta do eixo \\
\hline Força em X & $-1.576 \mathrm{~N}$ & Anel fixação da mola \\
\hline Força em Y & $-1.231 \mathrm{~N}$ & Anel fixação da mola \\
\hline
\end{tabular}

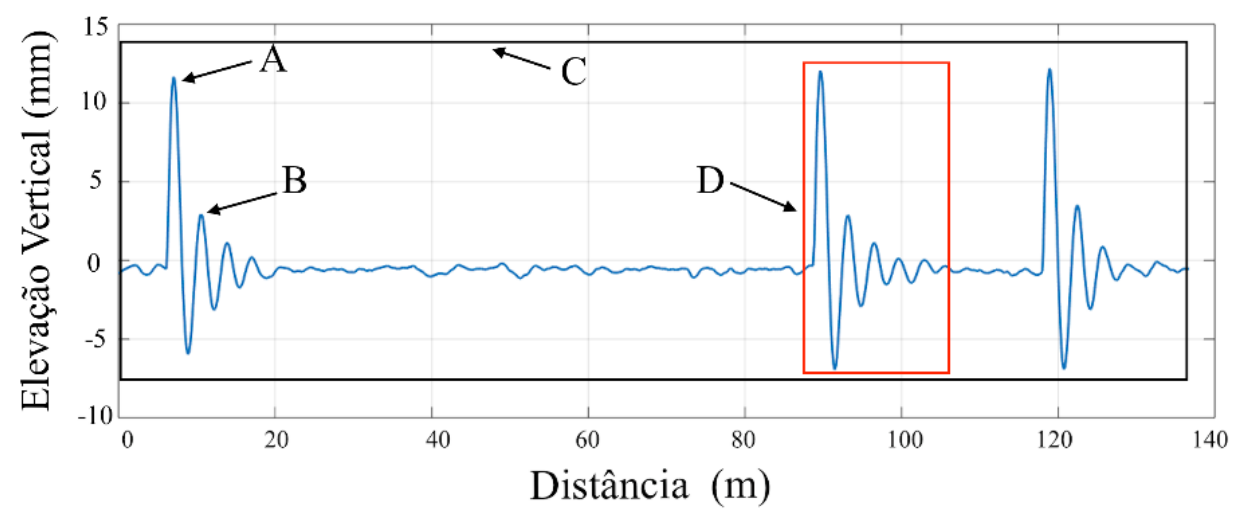

Figura 16 - Variáveis de saída do planejamento fatorial

Após avaliação, a combinação otimizada ocorre com mola pneumática de 3 bar, amortecedor com curva estabelecida e massa suspensa de $1 / 4$ de veículo igual a $200 \mathrm{~kg}$.

Fig. (17) mostra os deslocamentos verticais da massa suspensa e do pavimento com isso calculouse o valor $r m s$ de ambas as curvas para a determinação da transmissibilidade. $\mathrm{O}$ valor $r m s$ do pavimento é 0,6663, enquanto o do deslocamento da massa suspensa é 0,2192. Define-se a transmissibilidade pela razão entre a saída do sistema pela entrada. Neste caso, a transmissibilidade entre o pavimento e a massa suspensa, em termos de valor rms, é 0,3289 .

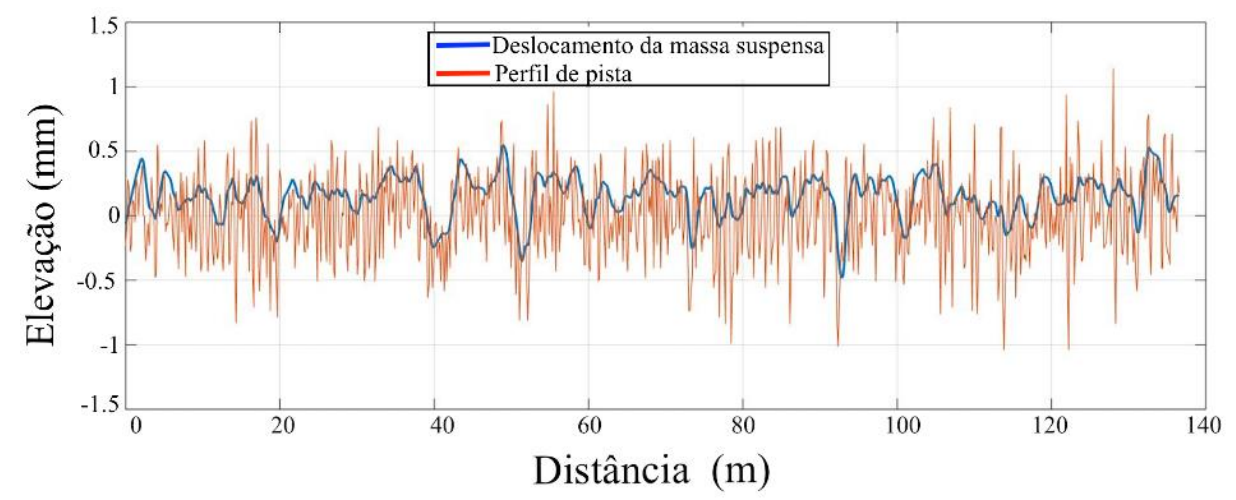

Figura 17 - Resposta da massa suspensa para o perfil de pista filtrado sem tachões 
Fig. (18) mostra a resposta da massa suspensa durante a passagem por uma irregularidade discreta (tachão). Nesse caso, a transmissibilidade é calculada tendo a altura do tachão como excitação de entrada, 50mm, e a resposta da massa suspensa será a excitação de saída, 11,21 mm. Portanto a transmissibilidade para esta verificação foi de 0,2242.

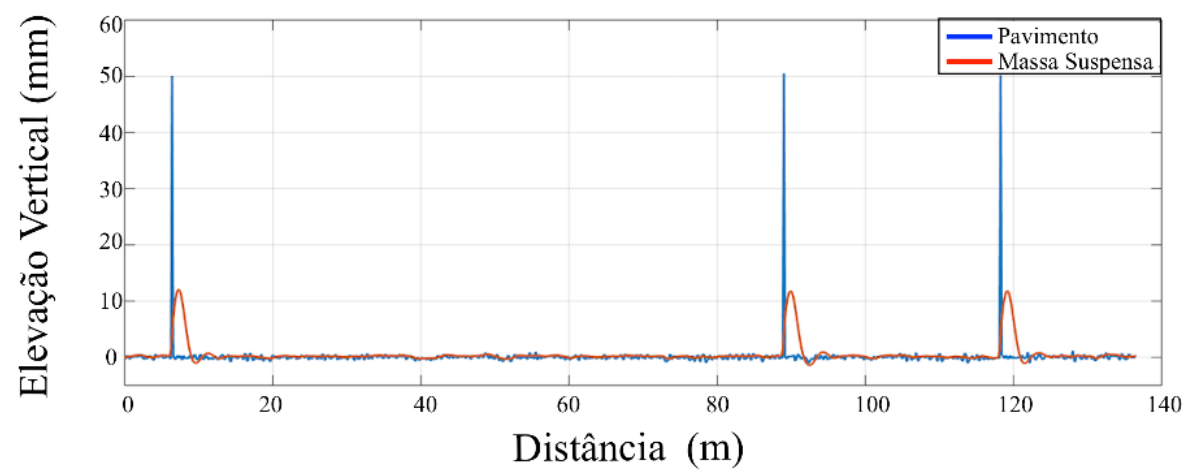

Figura 18 - Resposta da massa suspensa para o perfil de pista com tachões

\section{6 - Conclusão}

Os softwares multicorpos e elementos finitos mostraram-se ferramentas adequadas para a concepção de uma suspensão trailing arms aplicada a um perfilômetro inercial rebocável. A modelagem de 1/4 de suspensão mostrou-se adequada para a obtenção dos carregamentos em pontos pré-estabelecidos permitindo a verificação em elementos finitos, mostrando que o produto tem tensões abaixo da tensão de escoamento $\sigma_{\mathrm{Y}}$ do material. Foi possível verificar os deslocamentos da massa suspensa do modelo, que representa o módulo de medição do perfilômetro. A utilização de perfis de pista reais no estudo de ciclo de vida em fadiga de componentes, torna a análise mais fidedigna e mais adequada na determinação da segurança.

\section{Referência Bibliográfica}

Barella. R.M.. “ Contribuição para avaliação da irregularidade longitudinal de pavimento com perfilômetros inerciais".2008. 362 f.. Tese de doutorado. Universidade de São Paulo. São Paulo.

Côrtes, P.E.F., Desenvolvimento de acabamento rolled -on em aço inoxidável. 2009.126 f. Dissertação (Mestrado) - Universidade Federal de Minas Gerais. Belo Horizonte.

DNER, DNER PRO164/94: Calibração e controle de sistemas medidores de irregularidades de pavimento (Sistemas Integradores IPR-USP e Maysmeter). Brasília.1994.18 p.

DNIT, Sistema Nacional de Viação - SNV, 20015. Disponível no link (http://www.dnit.gov.br/sistema-nacional-de-viacao/snv-2014-1. Consultado em 26/06/2017.

Freitas JR., L.M.P. Estudo da dinâmica vertical de uma suspensão veicular do tipo Macpherson.2006. 139 f.. Dissertação de mestrado - Escola de engenharia de São Carlos - Universidade de São Paulo. São Paulo.

Gillespie, T.D. Fundamentals of vehicle dynamics. 1 ed. Michigan: Society of Automotive Engineers Inc. 
Montgomery, D.C. Design and analysis of experiments. 5o Edição. Arizona State University. 1997.

Rodrigues, L. de P. Proposição de perfis de pista baseado no efeito de fadiga de suspensão. 2015. Projeto de Graduação. Universidade de Brasília. Faculdade UnB Gama. Brasília.

Sayers, M.W.; Karamihas, S.M., The little book of profiling: Basic information about measuring and interpreting road profiles. University of Michigan.1998.

Vieira Neto, A. J. Metodologia de aperfeiçoamento de suspensões veiculares através de modelo virtual em ambiente multicorpos. 2011. 90 pg., Dissertação de mestrado - Escola de Engenharia de São Carlos. São Paulo. 